Cell Tissue Kinet. (1972) 5, 371-385.

\title{
SELF-RENEWAL OF COLONY FORMING UNITS (CFU) IN SERIAL BONE MARROW TRANSPLANTATION EXPERIMENTS
}

\author{
O. Vos AND M. J. A. S. Dolmans \\ Medical Biological Laboratory TNO, Lange Kleiweg 139, Rijswijk (Z.H.), \\ and Department of Cell Biology and Genetics, Rotterdam Medical Faculty'
}

(Received 17 February 1972; revision received 8 April 1972)

\begin{abstract}
The capacity of stem cells (CFU) for self-renewal was tested by transplanting normal bone marrow (primary transplantation) and bone marrow which had been subjected to one or two earlier transplantations (secondary and tertiary transplantation) into lethally irradiated syngeneic recipients. It was found that the capacity for self-renewal is diminished within the first weeks after one or more previous transplantations. This ability of stem cells recovered after a longer interval after the previous transplantation. The time required for this recovery depended upon the number of previous transplantations and amounted to more than 1 or 2 months after one or two transplantations respectively. Shortly after transplantation the $\mathrm{CFU} /$ nucleated cell ratio in bone marrow was below normal and its decrease was more pronounced when the bone marrow had been transplanted more often. An increase of the ratio towards normal values was observed in the course of one month after the last transplantation. Measurements of the spleen colony size after transplantation of normal and re-transplanted bone marrow indicated that CFUs from re-transplanted marrow gave slightly smaller spleen colonies than those of normal marrow.

It is concluded that the decreased self-renewal of stem cells shortly after previous transplantations is probably not due to a limitation in the number of normal mitoses they can perform, but to a loss of stem cells by transfer to the compartment of differentiating cells.
\end{abstract}

\section{INTRODUCTION}

When bone marrow is transplanted repeatedly from one lethally irradiated mouse into another, its capacity to keep the irradiated animal alive declines as the number of successive transplantations increases (Barnes, Ford \& Loutit, 1959; Van Bekkum \& Weyzen, 1961). In later experiments Cudkowicz et al. (1964), Siminovitch, Till \& McCulloch (1964) and

Correspondence: Dr O. Vos, Department of Cell Biology and Genetics, Rotterdam Medical Faculty, Dr. Molewaterplein 50, Rotterdam, The Netherlands. 
Lajtha \& Schofield (1971) studied this decline phenomenon with the spleen colony technique. The decline of the self-renewal capacity of stem cells in successive bone marrow transplantations and the resulting failure to graft bone marrow serially is a phenomenon which may be comparable with the impossibility to carry out serial transplantations of other tissues such as mammary tissue (Daniel et al., 1968; Daniel \& Young, 1971) and skin (Krohn, 1962).

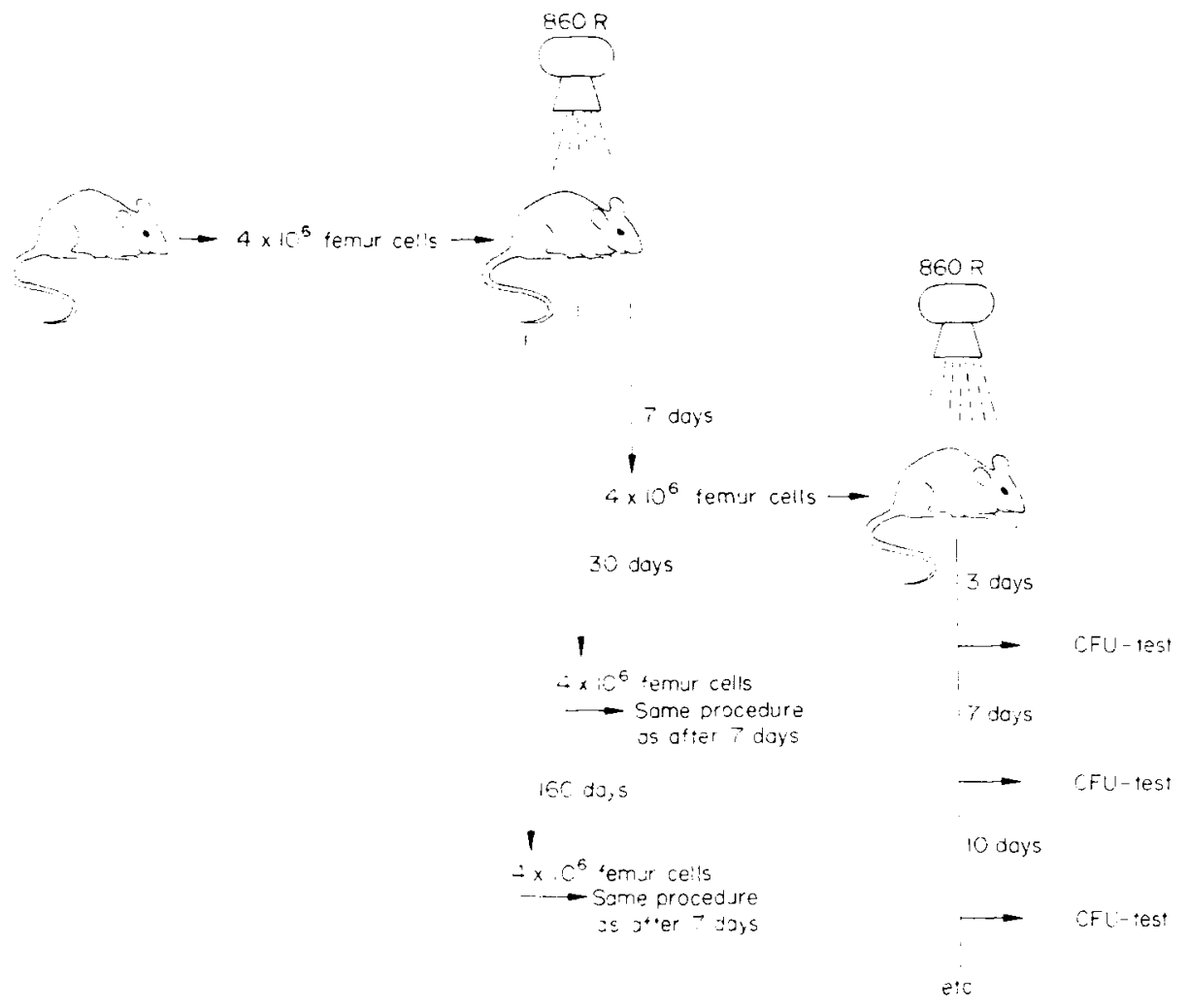

FIG. 1. Scheme of experiments in which CFU multiplication was studied after a secondary bone marrow transplantation performed at various times after the first transplantation.

In all cases it seems that proliferation of cells in normal tissues is limited. Hayflick \& Moorhead (1961) and Hayflick (1965) observed that human diploid cells have a limited lifetime in citro. The possibility that a limitation of the number of mitoses in normal diploid cells is playing a role as an ageing process at the cellular level has been discussed by other authors (Kay, 1965: Holeckova \& Cristofalo, 1970).

In the present paper the kinetics of the self-renewal of haemopoietic stem cells in serial transplantation experiments has been studied and a possible relationship between the limitation of serial grafting and a limited number of mitoses of normal diploid cells will be discussed. 


\section{MATERIALS AND METHODS}

$(\mathrm{C} 57 \mathrm{BL} \times \mathrm{CBA}) \mathrm{F}_{1}$ male mice, 10-16 weeks old, weighing 22-32 g, were used. Recipients were irradiated with a lethal dose of X-rays ( $825 \mathrm{rad}$ from a Philips $250 \mathrm{kV}$ X-ray machine).

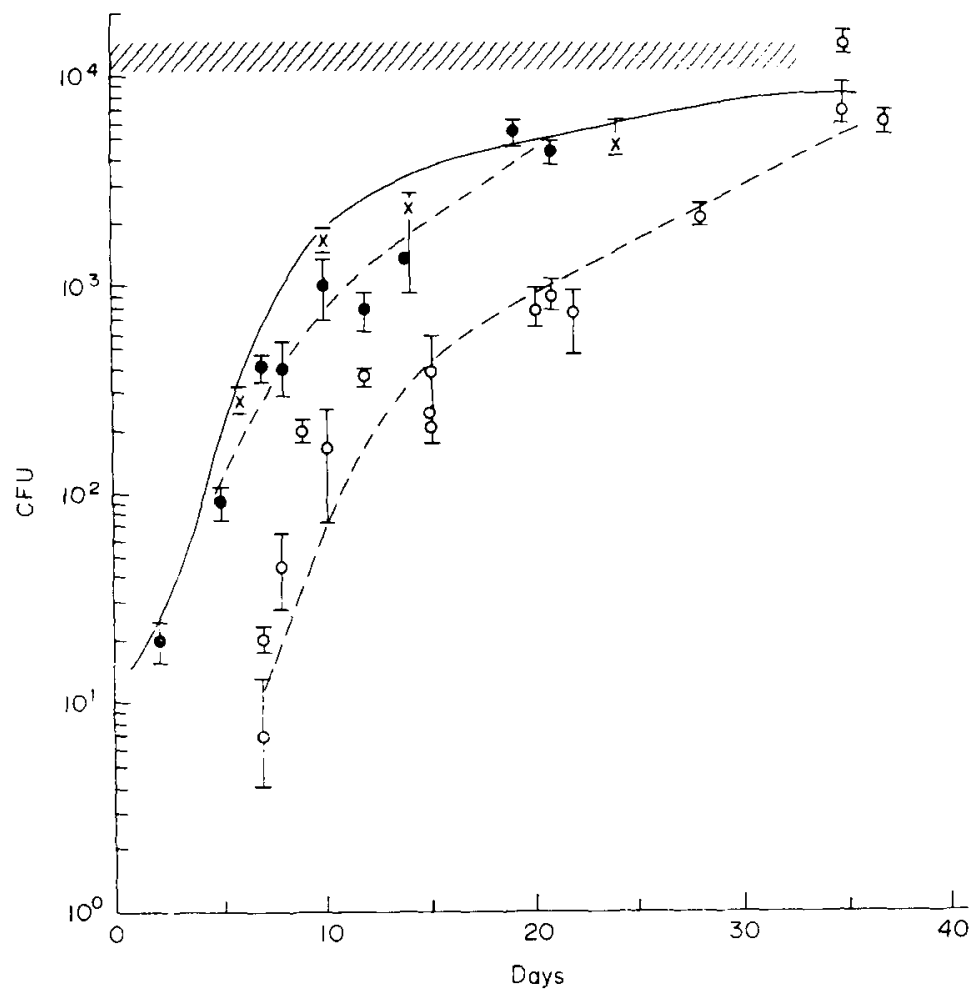

FIG. 2. CFU growth curves in femoral bone marrow after primary or secondary transplantation of $4 \times 10^{6}$ bone marrow cells; - - primary transplantation (the experimental points on which this curve is based are presented in Fig. 4); $\odot$, secondary transplantation 7 days after first transplantation; $\bullet$, secondary transplantation 30 days after first transplantation; $x$, secondary transplantation 160 days after first transplantation. The hatched area shows the level of CFU in normal mice. Curves were drawn by eye. $95 \%$ confidence limits are indicated.

The radiation conditions are described elsewhere (Vos, 1972a). Bone marrow and spleen cell suspensions were prepared as described earlier (Vos, 1967). Cell suspensions were injected into the tail vein within a few hours after irradiation. The number of colony forming units (CFU) in femur and spleen was assessed by injecting fractions of femoral bone marrow and spleen cell suspensions into lethally irradiated syngeneic mice and counting the spleen colonies 8 days later. Suspensions were always made from at least three donor mice and $0.5 \mathrm{ml}$ of the cell suspension was injected per recipient. Spleens were fixed in Bouin's solution, and colonies were counted using the low power objective of a stereo-microscope. After pilot experiments the fractions of the spleen and bone marrow suspensions could be chosen in 
such a way that the number of colonies per spleen varied between one and twenty. Of each cell suspension three dilutions were made, each differing from the other by a factor 2 in cell concentration. Each dilution was injected into ten mice. Hence the number of CFU per spleen or femur was calculated from colony counts in thirty mice. The number of viable nucleated cells in each cell suspension was counted in a Bürker haemocytometer, using the

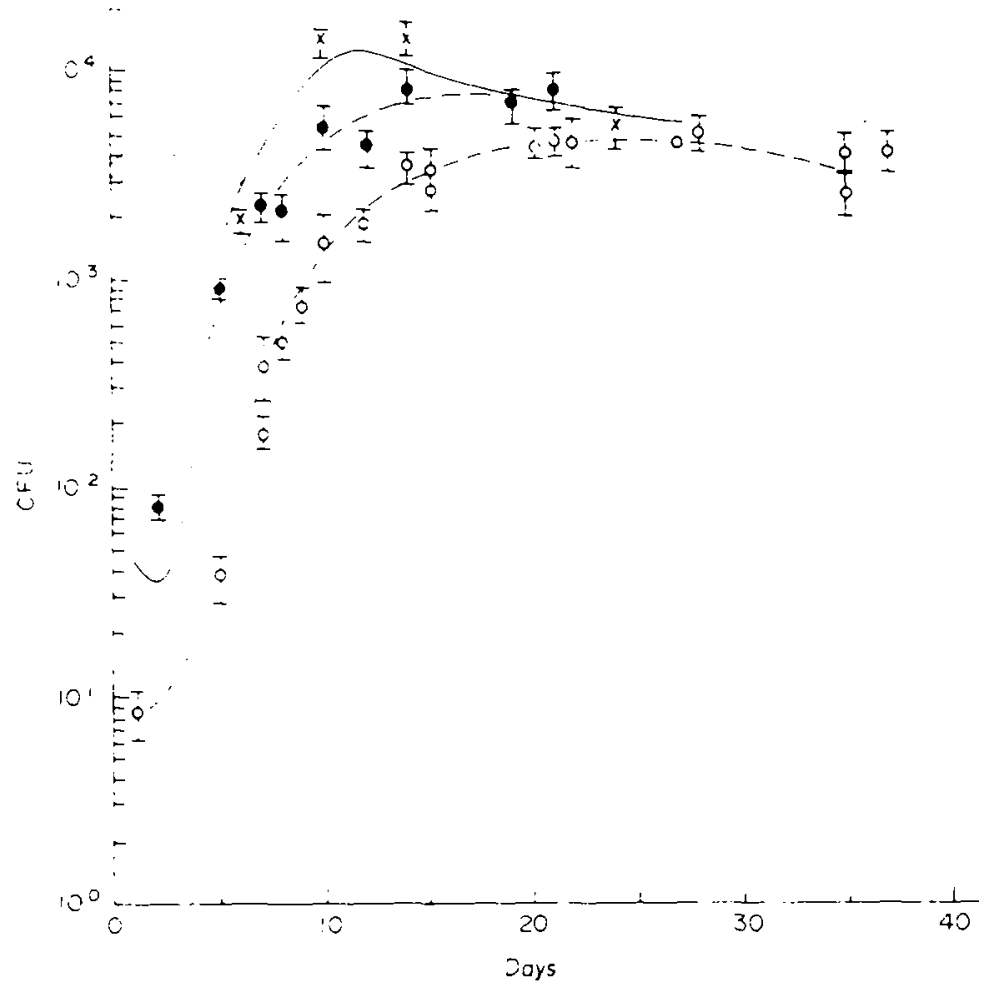

Fig. 3. CFU growth curves in spleen after primary and secondary transplantation of $4 \times 10^{6}$ bone marrow cells. The data are from the same experiments as presented in Fig. 2; symbols also as in Fig. 2. The growth curve after primary transplantation is based on points presented in Fig. 5. The curves were drawn by eye.

eosin dye exclusion test for viability. The percentage of CFU per $10^{6}$ viable nucleated cells could be calculated from these data. The CFU-multiplication curve in femoral bone marrow and spleen was determined with the method of periodic sampling (McCulloch \& Till, 1964): mice were sacrificed various times after irradiation and bone marrow transplantation and the CFU content in femoral bone marrow and spleen was assessed with the exogenous spleen colony technique. The experiments were repeated and the data obtained in two or more experiments are presented in the figures.

The size of the fixed spleen colonies was measured under the microscope (magnification 10 times) with an ocular micrometer. which was calibrated with an objective micrometer. 


\section{RESULTS}

In the first set of experiments the increase of the number of CFUs (growth curve) after transplantation of normal bone marrow cells (primary transplantation) was compared with the corresponding growth curve after transplantation of bone marrow cells from syngeneic chimaeras (secondary transplantation). The CFU growth curves in femoral bone marrow

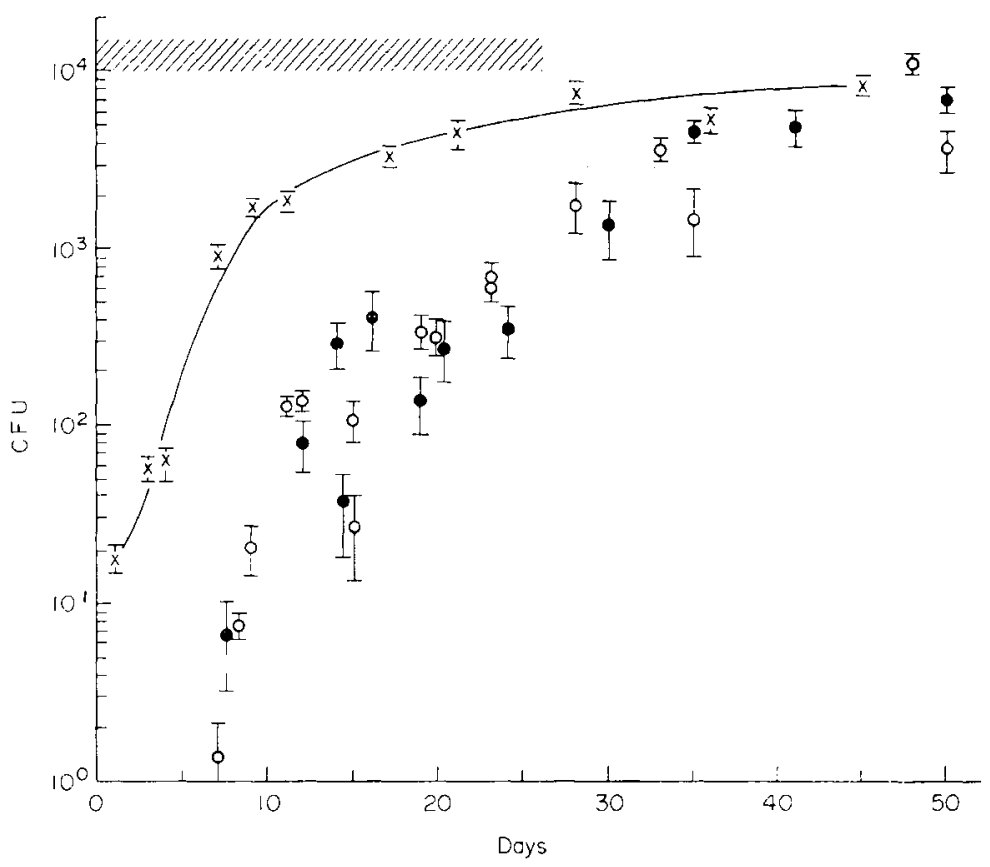

Fig. 4. CFU multiplication in femoral bone marrow after primary or tertiary transplantation of $4 \times 10^{6}$ bone marrow cells; $\times$, primary transplantation; $O$, tertiary transplantation 14 days after second transplantation; $\bullet$, tertiary transplantation 21 days after second transplantation. The hatched area shows the level of CFUs in normal mice. The curve is drawn by eye. $95 \%$ confidence limits are indicated.

and spleen were investigated. The secondary transplantation was performed 7,30 or 160 days after the primary transplantation. In each case the growth curve of CFUs from $4 \times 10^{6}$ transplanted bone marrow cells was studied. A scheme of the experiments is depicted in Fig. 1. The growth curves in femoral bone marrow and spleen are presented in Figs. 2 and 3. The best fitting curves have been drawn. Due to the fact that the statistical variation between different experiments was somewhat greater than the statistical variation within one experiment, there is some scattering of the points at both sides of the curves which have been drawn. When in the secondary transplantation experiment the cell suspension was prepared 7 days after the first transplantation the growth curve in bone marrow and spleen commenced at a lower level than in the primary transplantation experiment. The accurate starting point fell below the level which could be determined. As will be described later (Fig. 8), this 
difference is probably due to a lower fraction of CFUs per number of nucleated cells in the cell suspension injected. Thirty days after the first transplantation the difference in starting level was no longer observed. In the spleen the overshoot of CFU observed between the tenth and twentieth day after a primary transplantation was absent or less prominent in case of a secondary transplantation when the interval between the first and second transplantation was 7 days. When this interval increased up to 30 days the difference between the growth curve of primarily and secondarily transplanted CFUs became less marked both in bone marrow and spleen and when the interval was 160 days no difference could be observed.

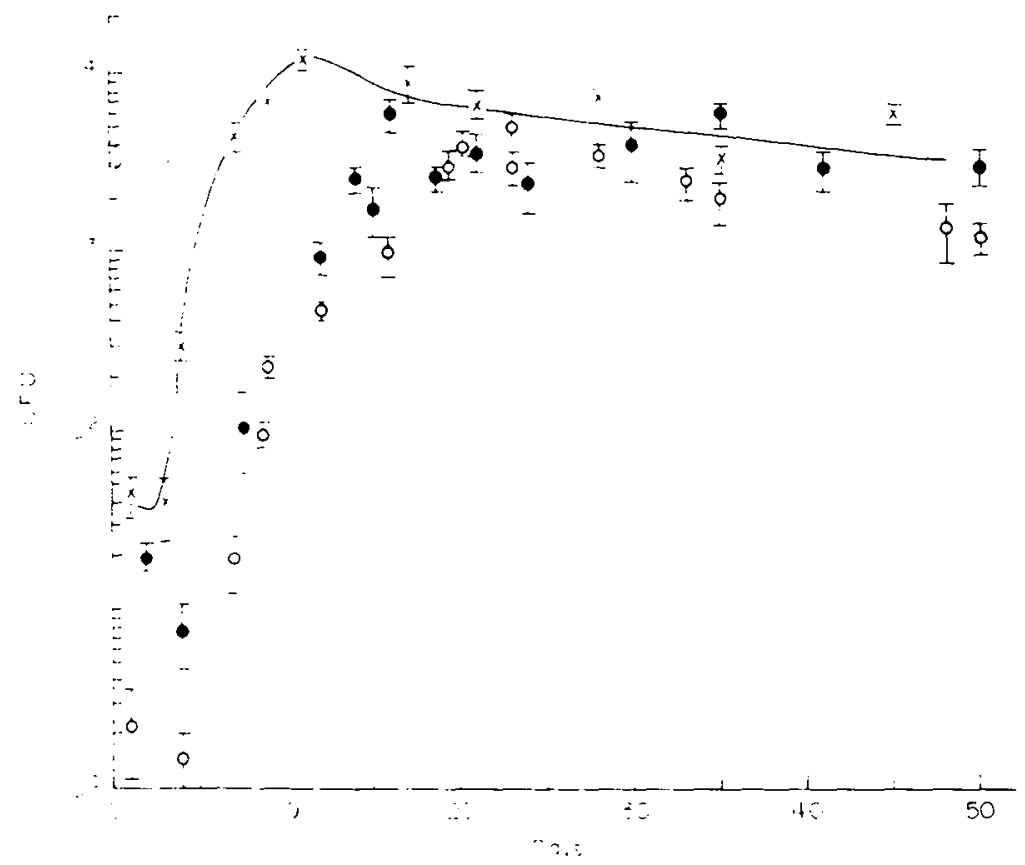

FiG. 5. CFU multiplication in spleen after primary and tertiary transplantation of $4 \times 10^{6}$ bone marrow cells. The data are from the same experiments as presented in Fig. 4; symbols also as in Fig. 4.

In the next set of experiments the growth pattern of CFUs in tertiary transplanted bone marrow was studied. In these experiments the second transplantation was performed 7 days after the first and the third transplantation was carried out $14,21,37$ or 61 days after the second transplantation. In every passage $4 \times 10^{6}$ bone marrow cells were grafted. The growth rates in femoral bone marrow and spleen are presented in Figs. 4-7. Although there is a wide scattering between individual points the results indicate that the increases of CFUs, when a tertiary transplantation was performed 14 or 21 days after the second transplantation. in the bone marrow (Fig. 4) and in the spleen (Fig. 5) were generally comparable to those after performance of the secondary transplantation 7 days after the first. However, the level on which proliferation commenced, was lower and the growth rate slower (compare Fig. 4 with Fig. 2 and Fig. 5 with Fig. 3) than after a second transplantation. When the interval between the second and the third transplantation was extended the growth pattern became 
again more like that after a primary transplantation. On the sixty-first day after the second transplantation it had practically regained the growth potential of bone marrow in the first transplantation (Figs. 6 and 7).

The ratio between the number of CFUs and the number of nucleated cells in femoral bone marrow and in the spleen at various times after a first, second or third transplantation is

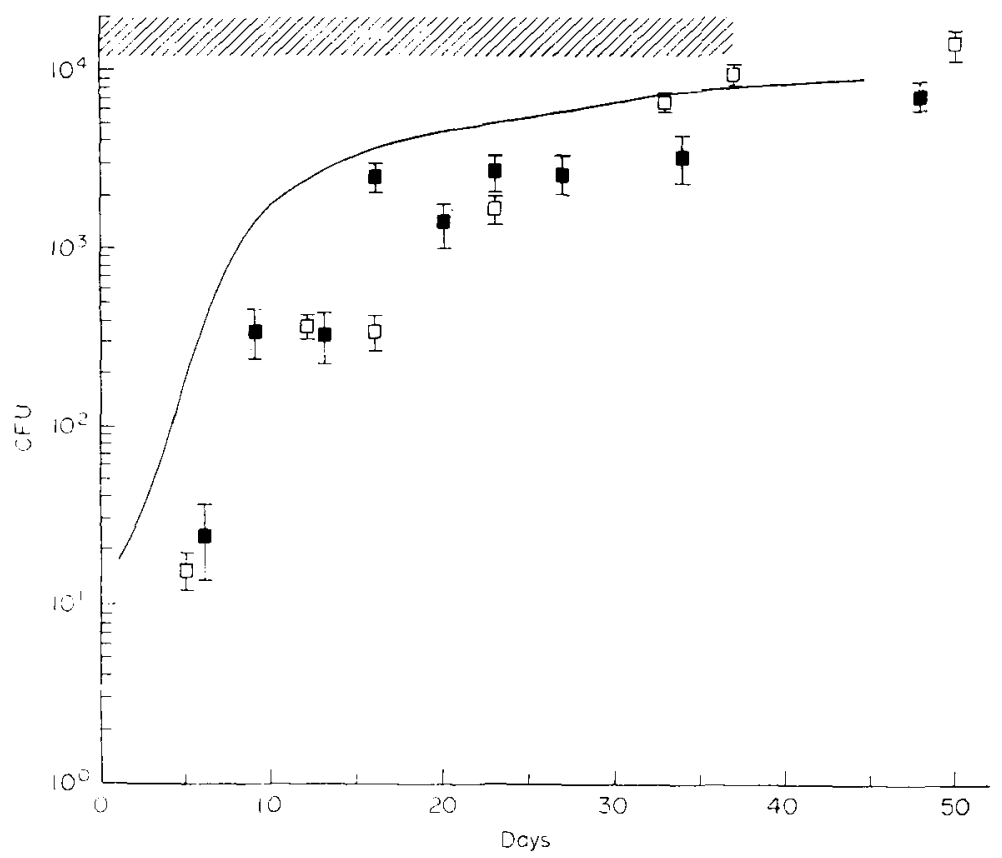

Fig. 6. CFU multiplication in femoral bone marrow after primary or tertiary transplantation of $4 \times 10^{6}$ bone marrow cells; — - primary transplantation (line is the same as in Fig. 4); $\square$, tertiary transplantation 37 days after second transplantation; $\boldsymbol{m}$, tertiary transplantation 61 days after second transplantation. The hatched area shows the level of CFUs in normal mice. $95 \%$ confidence limits are indicated.

expressed in Figs. 8 and 9. After a primary transplantation this ratio remains in the bone marrow below normal until about 30 days after transplantation. After a secondary and a tertiary transplantation the ratio during the first $\mathbf{2 0}$ days is lower than after a primary transplantation. Still the normal level is attained between about 30 and 40 days. In the spleen the ratio may begin below the normal level but it is already above this level on the third day after transplantation, it increases further until the tenth day and then it slowly decreases towards the normal level. The overshoot is concomitant with the replacement of the lymphoid cells by haemopoietic cells that occurs after bone marrow transplantation in irradiated mice. After a secondary transplantation the ratio in the spleen remains below normal until the fifteenth day and after the tertiary transplantation the normal level is not attained before the twentieth day. The overshoot in case of a secondary and tertiary transplantation comes later and is less pronounced than after a primary transplantation. 


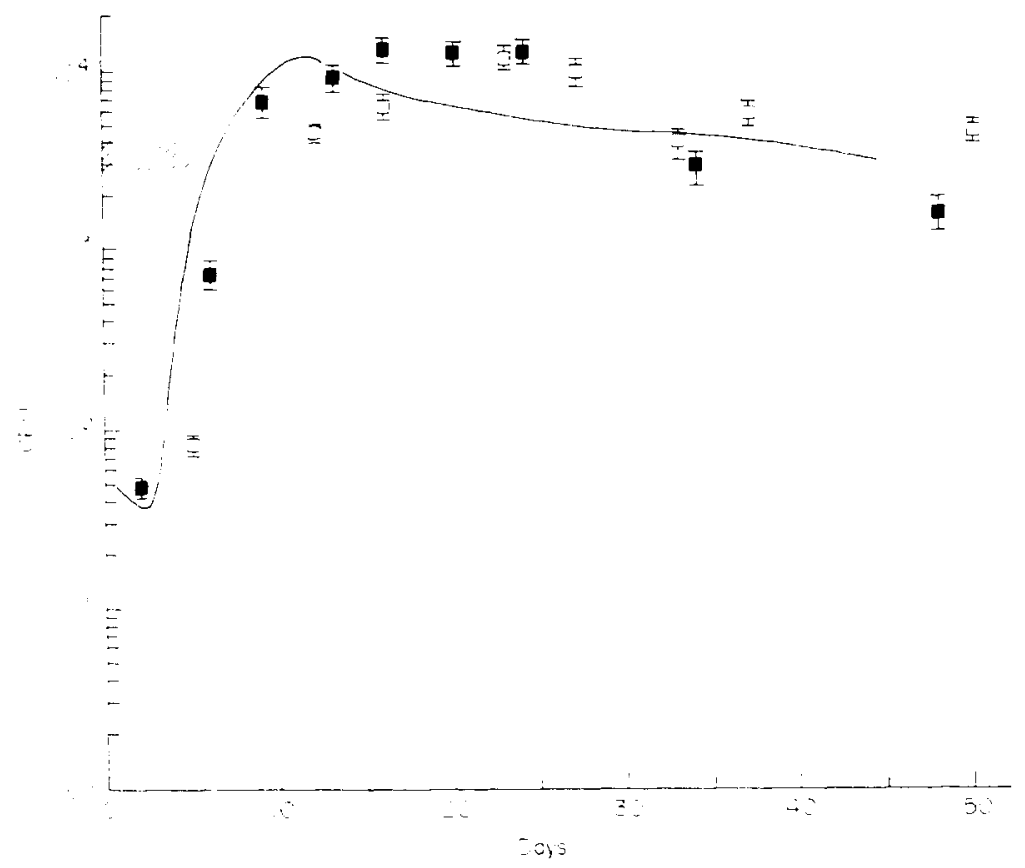

FIG. 7. CFU multiplication in spleen after primary or tertiary transplantation of $4 \times 10^{6}$ bone marrow cells. The data are from the same experiments as presented in Fig. 6; symbols also as in Fig. 6.

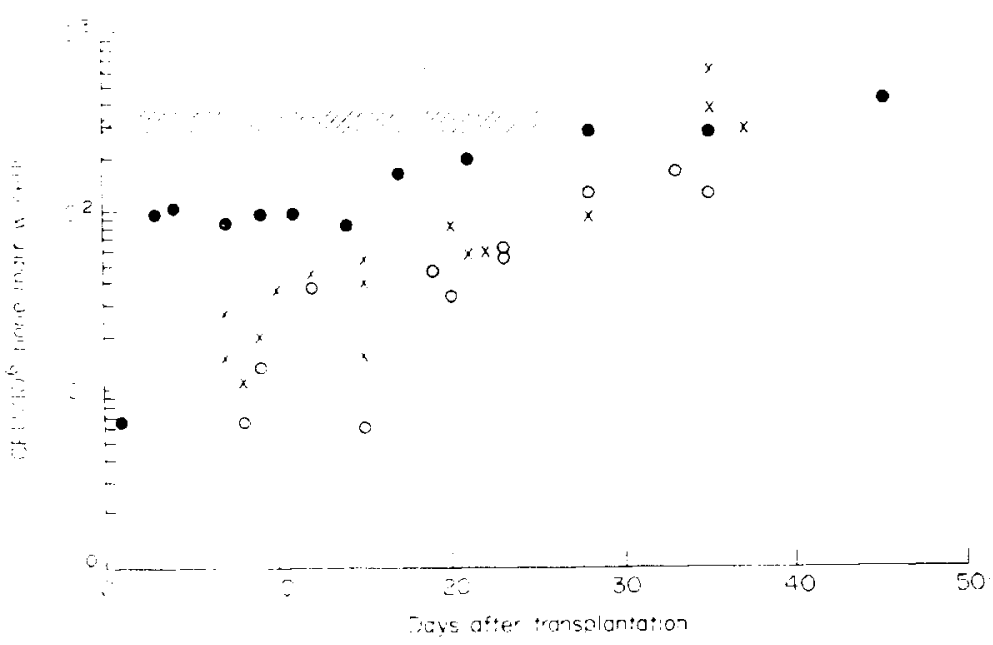

FIG. 8. Number of CFUs (assayed by the exogenous spleen colony technique) per million nucleated cells in femoral bone marrow at various times after transplantation of normal bone marrow (primary transplantation), - secondary transplantation (interval between first and second transplantation 7 days), $x$; tertiary transplantation (first and second interval between transplantations 7 and 14 days respectively), :; hatched area: level in normal mice. 
In Table 1 measurements of the size of spleen colonies 8 days after primary and tertiary transplantation are presented. The differences are small but the following conclusions can be drawn: (a) when the average number of colonies per spleen becomes greater, due to a greater number of CFU injected, the average size of the spleen colonies also increases;

TABLE 1. Effect of repeated transplantations on the size of spleen colonies

\begin{tabular}{|c|c|c|c|c|c|}
\hline \multicolumn{3}{|c|}{ Primary transplantation } & \multicolumn{3}{|c|}{ Tertiary transplantation* } \\
\hline $\begin{array}{l}\text { Fraction } \\
\text { grafted }\end{array}$ & $\begin{array}{l}\text { Average number } \\
\text { of colonies } \\
\text { per spleen }\end{array}$ & $\begin{array}{l}\text { Diameter of colonies } \\
(\mathrm{mm})(\text { arithmetic } \\
\text { mean } \pm \text { S.E. })\end{array}$ & $\begin{array}{c}\text { Fraction } \\
\text { grafted }\end{array}$ & $\begin{array}{l}\text { Average number } \\
\text { of colonies } \\
\text { per spleen }\end{array}$ & $\begin{array}{l}\text { Diameter of colonies } \\
(\mathrm{mm})(\text { arithmetic } \\
\text { mean } \pm \text { S.E. })\end{array}$ \\
\hline \multicolumn{6}{|c|}{ FEMORAL BONE MARROW } \\
\hline $1 / 1000$ & $11 \cdot 4$ & $1.261( \pm 0.043)$ & $1 / 50$ & 8.9 & $0.998( \pm 0.037)$ \\
\hline $1 / 2000$ & $6 \cdot 3$ & $1 \cdot 124( \pm 0.042)$ & $1 / 100$ & $5 \cdot 4$ & $0.822( \pm 0.031)$ \\
\hline $1 / 4000$ & $5 \cdot 1$ & $1.018( \pm 0.038)$ & $1 / 200$ & $3 \cdot 1$ & $0.835( \pm 0.043)$ \\
\hline \multicolumn{6}{|l|}{ SPLEEN } \\
\hline $1 / 500$ & $8 \cdot 3$ & $1 \cdot 107( \pm 0.035)$ & $1 / 500$ & $6 \cdot 3$ & $0.908( \pm 0.030)$ \\
\hline $1 / 1000$ & $5 \cdot 0$ & $1.000( \pm 0.040)$ & $1 / 1000$ & $2 \cdot 4$ & $0.865( \pm 0.042)$ \\
\hline $1 / 2000$ & $2 \cdot 6$ & $0.922( \pm 0.059)$ & $1 / 2000$ & 1.9 & $0.841( \pm 0.039)$ \\
\hline
\end{tabular}

* The first transplantation was a transplantation of $4 \times 10^{6}$ femoral bone marrow cells followed by a second transplantation of the same number of bone marrow cells 7 days later; the last transplantation was either a bone marrow or a spleen transplantation 14 days after the previous transplantation.

(b) when numbers of colonies per spleen in case of a primary and a tertiary transplantation are similar the diameters of the former appear to be larger than the latter.

In the experiments described so far the number of nucleated bone marrow cells transplanted in the primary, secondary and tertiary transplantation was kept constant. Since the CFU/nucleated cells ratio varied (see Fig. 8) the number of CFUs must have also been different in subsequent transplantations.

In order to compare the growth curves when the same numbers of CFUs are transplanted in a primary and a tertiary transplantation a serial transplantation experiment was performed in which the number of transplanted CFU was kept constant. At the moment of transplantation the CFU ratio is not known exactly and it had to be estimated on basis of the data presented in Fig. 8. The real number of CFUs per $10^{4}$ nucleated cells in each transplantation was determined with the spleen colony technique, so that the number of transplanted CFUs could be calculated subsequently. The number of transplanted bone marrow cells and CFUs in this experiment are presented in Table 2. The CFU growth curve after the third transplantation is presented in Figs. 10 and 11. The results show that in the bone marrow as well as in the spleen the growth rate lies between the curve after a tertiary transplantation of $4 \times 10^{6}$ bone marrow cells and that after a primary transplantation. The slope is similar to that after tertiary transplantation of $4 \times 10^{6}$ cells. In the spleen practically no overshoot was found, although the level on which the proliferation started was the same as that after a primary transplantation of $4 \times 10^{6}$ marrow cells. 


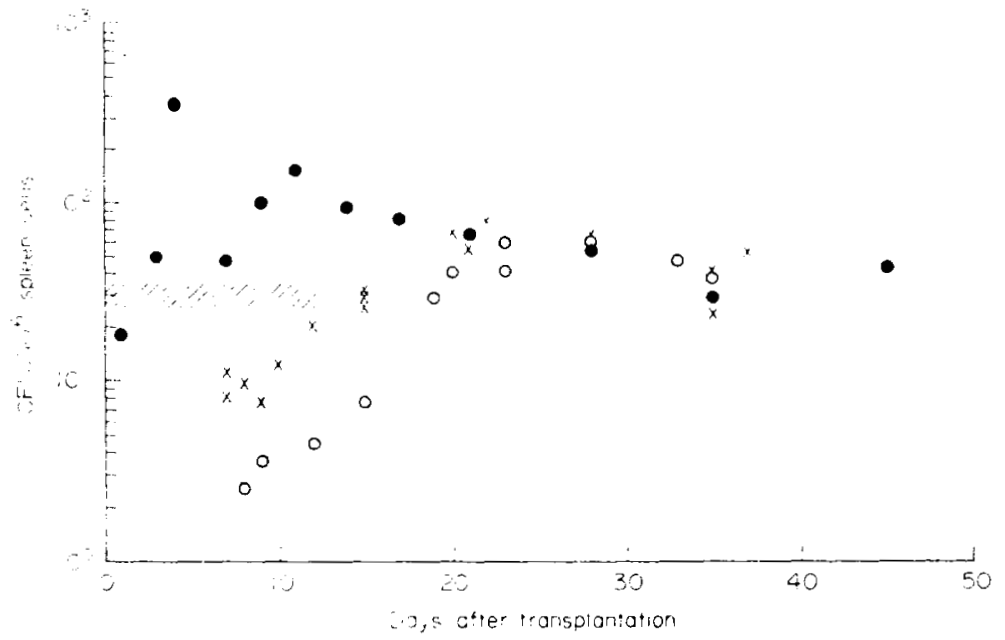

FIG. 9. Number of CFUs.per million nucleated cells in the spleen after primary, secondary and tertiary transplantation. For further information and symbols see Fig. 8.

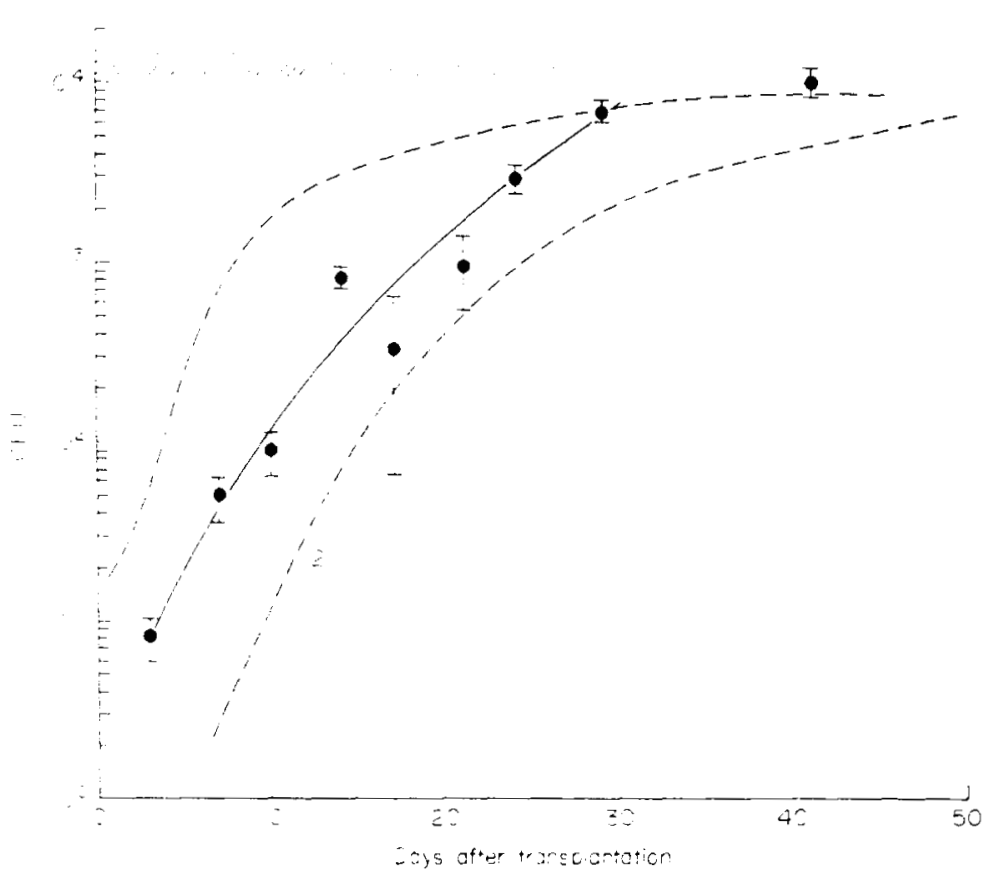

FIG. 10. CFU growth curve in femoral bone marrow after tertiary transplantation of about 2200-2900 CFUs (see Table 2). $95^{\circ} \%$ confidence limits are indicated. The curve is drawn by eye. The broken line no. 1 represents the growth curve after primary transplantation of $4 \times 10^{6}$ bone marrow cells; the broken line no. 2 represents the growth curve after tertiary transplantation of an experiment in which the intervals between the transplantations were the same as in this experiment, the number of transplanted nucleated calls was kept constant on $4 \times 10^{6}$ (see Fig. 4). The hatched area represents the level of CFUs in normal spleens. 
TABLE 2. Serial bone marrow transplantation experiment in which the number of transplanted CFU was tried to be kept constant

Interval after previous No. of transplanted

No. of

transplantation (days) bone marrow cells $\left(10^{-6}\right)$ transplanted CFU

\begin{tabular}{lrrr}
\hline First transplantation & - & 4 & 2220 \\
Second transplantation & 7 & 10 & 2595 \\
Third transplantation & 14 & 30 & 2880
\end{tabular}

In the last experiment it was investigated whether one transfer of bone marrow derived CFUs through the spleen altered their growth rate in spleen or bone marrow at the following transplantation. $4 \times 10^{6}$ bone marrow cells (about $1600 \mathrm{CFU}$ ) were grafted in the first transplantation and $4 \times 10^{6}$ marrow cells (about $400 \mathrm{CFU}$ ) or $4 \times 10^{6}$ spleen cells (about $400 \mathrm{CFU}$ ) were grafted in the secondary transplantation. The growth curves after grafting 400 splenic CFU in the secondary transplantation are depicted in Fig. 12. The curves can be compared with the corresponding curves after one passage through bone marrow. It will

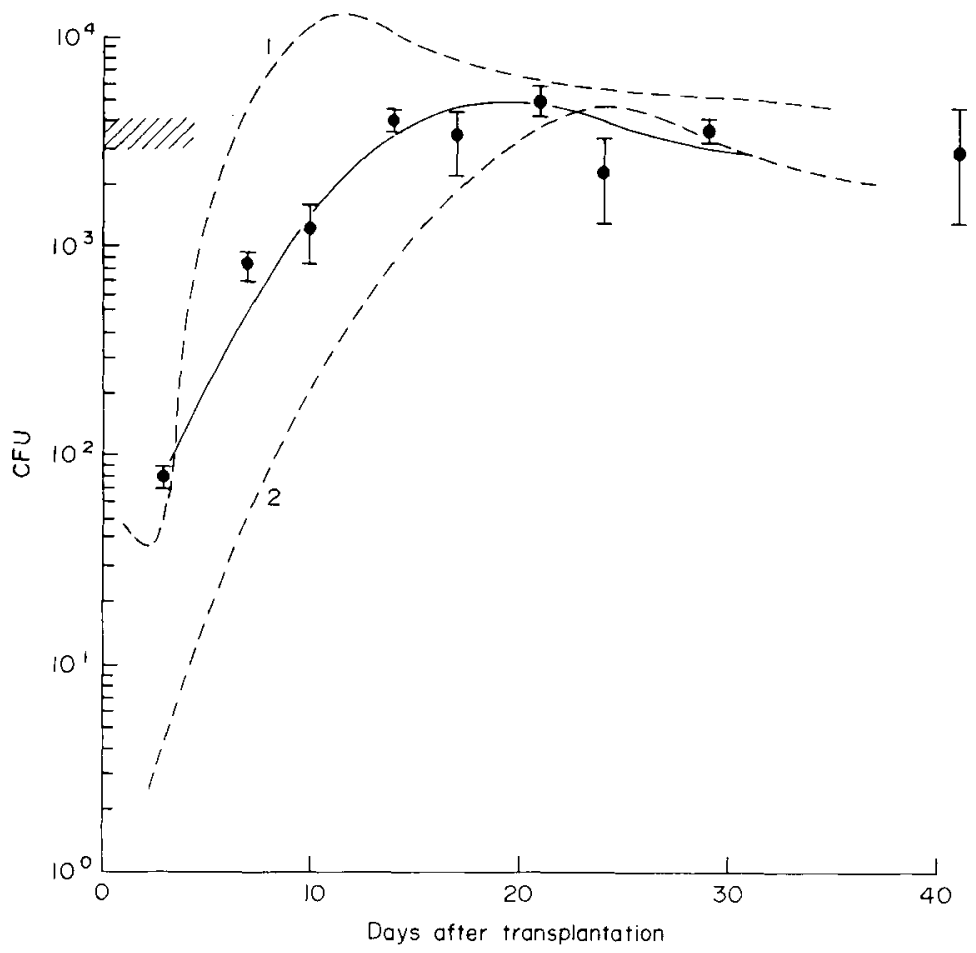

FIG. 11. CFU growth curve in the spleen after tertiary transplantation of about 2200-2900 CFUs (see Table 2). For further explanation see Fig. 10. 


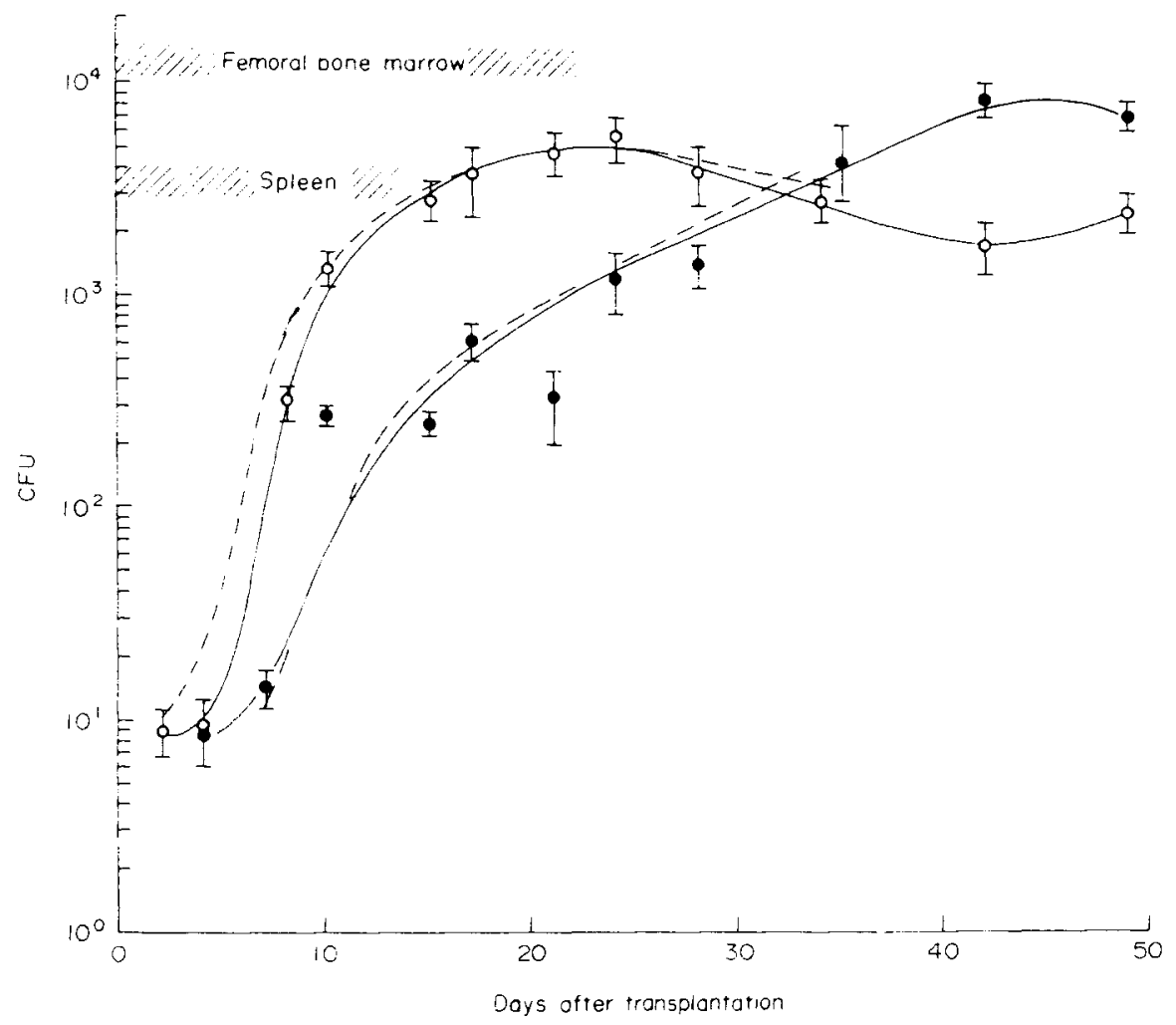

FIG. 12. CFU growth curves in femoral bone marrow and spleen after one passage through the spleen (solid lines): interval between first and second transplantation 7 days. $\bullet$, Number of CFU in bone marrow: number of CFU in spleen; $95 \%$ confidence limits are indicated. The hatched areas show the normal levels of CFU in femoral bone marrow and spleen. The broken lines are representing the growth curves in bone marrow and spleen after one passage through the bone marrow and are taken from Figs. 2 and 3.

be clear that after one passage through the spleen the subsequent growth curves of CFUs in bone marrow and spleen is the same as after one passage through femoral bone marrow.

\section{DISCUSSION}

The starting level of the growth curve in the spleen after the secondary transplantation (interval between the two transplantations 7 days) was $4-5$ times lower than after a primary transplantation (Fig. 3). It seems reasonable to explain this difference by the lower CFU/ nucleated cell ratio in the former cell suspension (see Fig. 8) which is also about a factor 4 lower than in a normal bone marrow suspension. In bone marrow the starting level of the growth curve (Fig. 2) was not determined in case of secondary transplantation since it was 
too low for an accurate determination, and consequently no conclusions can be drawn. After a tertiary transplantation (and short intervals between the transplantations, Figs. 4 and 5) the starting levels in both spleen and bone marrow were too low for an accurate assay; however, the results do not seem to be in disagreement with what could be expected on base of the number of transplanted cells and the CFU/nucleated cell ratio. The growth curves of CFUs from bone marrow obtained in secondary and tertiary transplantation experiments recover towards that of normal bone marrow (primary transplantation) when the time after the last transplantation is extended (Figs. 2, 3,6 and 7). This can partly be explained by the increase of the $\mathrm{CFU} /$ nucleated cell ratio in the course of time after the last transplantation. As a result the number of transplanted CFUs in $4 \times 10^{6}$ bone marrow cells becomes similar to that in $4 \times 10^{6}$ normal bone marrow cells. However, in addition to the increasing number of transplanted CFU and consequently the higher starting level of the growth curve, there is also a change in the slope of the growth curve. Shortly after one or two transplantations the slope is less steep than when normal marrow is investigated. Furthermore in the spleen the overshoot is smaller or absent. Both observations can be explained as a diminished capacity for multiplication after successive transplantations. The CFU growth curves regain their normal slope and in the spleen the overshoot recovers when the time after the last transplantation is extended. The experiment in which a few successive transplantations with a constant number of CFUs (Figs. 10 and 11) was performed, and in which a less steep slope of the CFU growth curve was found show that CFUs from bone marrow which has been subjected to a period of excessive proliferation shortly before the test have changed their doubling time. Previously published data (Vos, 1972a, b), although less conclusively, also suggests that self-renewal of CFUs temporarily slows down during a period of excessive proliferation.

The size of spleen colonies after primary and tertiary transplantation (Table 1) further suggests that on the average the whole progeny of a stem cell from a population which has been subjected to an excessive proliferation during the preceding weeks has a smaller capacity for proliferation than the progeny of a stem cell which comes from a steady state population. Although the differences we found are small and of limited value they are in agreement with the finding of Blomgren (1971) who described that the size of spleen colonies from bone marrow taken shortly after one or a few successive $X$-irradiations is smaller than that from normal marrow. When he took bone marrow at a later time (2-3 weeks) after the last irradiation the colony size was the same as that of normal bone marrow. This recovery may be analogous to the recovery of the CFU growth rate at a later time after one or two transplantations, which we described in this paper. Blomgren's finding that the time needed for recovery after a multiple irradiation is longer than after a single irradiation may also be comparable with our observation that it takes more time to recover after two transplantations than after one. A technical point in our spleen size measurements which needs to be discussed is that the diameter of the colony depended upon the average number of spleen colonies counted per spleen. A logical explanation for this finding seems that in spleens with a greater number of colonies some confluent clones of two CFUs are counted as one colony, or that smaller colonies are missed progressively when the number of colonies increases. For this reason the diameter of colonies in spleens with the same average colony number should be used when a comparison of the size is to be made. When this is done the average size of colonies from normal marrow is still larger than from transplanted marrow. Schofield \& Lajtha (1969) described that in lethally irradiated mice grafted with different numbers of 
syngeneic bone marrow cells, spleen colonies in mice grafted with the greatest number of cells contained less cells than the colonies in spleens of mice grafted with a smaller number of bone marrow cells. This difference is most evident 12 and 15 days after grafting, but it already exists on the eighth day. These authors speculate that 'the growth of colonies from the larger grafts is restricted because of the available space in the spleen'. Within the graft size variation we used (maximum difference was a factor of 4 ), we were unable to confirm the observation of Schofield \& Lajtha.

In the present experiments all recipients were irradiated with an X-ray dose that was at least 50 rad above the lethal dose. This makes it improbable that in serial transplantation experiments recovery of the recipient's own haemopoietic tissue contributed to the population of transplanted cells and that in this way it caused a recovery of the CFU growth rate. However, in a long series of transplantation experiments the absence of a contribution of recipient cells to the repopulation has to be proven by using chromosome markers in the donor bone marrow suspension. We did not use such markers and therefore the studies were not extended beyond three passages. In earlier investigations (Vos, 1972b) we showed that a similar recovery of the growth ability of CFUs as described here, is found after three sublethal irradiations. In such conditions only the original populations of the irradiated mouse can be involved and this supports our conclusion that recovery of the growth rate of CFU occurs when they are given time to restore the steady state condition after a period of excessive proliferation.

The reason why the growth potential of CFU decreases during a period of intensive proliferation is not clear. An explanation in terms of a limited number of mitoses for normal diploid cells as suggested by Hayflick \& Moorhead (1961) for cells in culture and by Daniel \& Young (1971) for normal mammary cells cannot fully be excluded but seems improbable. The recovery of the growth rate is difficult to explain by this theory. A better tenable explanation seems that during a period of excessive proliferation CFUs are lost to a more differentiated cell compartment and are losing their capacity for unlimited proliferation. This is in agreement with a suggestion of Bennett \& Cudkowicz (1968) that 'the decline of serially transplanted marrow may well find its explanation in rapid conversion of stem cells to precursor cells owing to demands for differentiated cells'. During a period of steady state the compartment of CFUs (for a large proportion in $\mathrm{G}_{0}$ phase) is restored. If such changes take place, it would imply that the self-renewal probability of stem cells which Vogel, Niewisch \& Matioli (1970) found to be constant during the sixth and fourteenth day of colony development alters under more excessive conditions such as serial transplantation.

In the last experiment it was shown that the environment of the spleen or bone marrow during one passage has no effect on the subsequent ability of CFUs for self-renewal. Schofield (1970) observed that CFUs from bone marrow and spleen had different doubling times and that certain differences were maintained when the cells were passed through the spleen. Our results do not need to be in conflict with Schofield's data, because they only show that a passage through the spleen does not induce other changes in growth characteristics of bone marrow CFUs than one passage through bone marrow. Furthermore we found that one passage through the spleen as well as one passage through bone marrow slows down the growth rate of bone marrow CFUs. This last finding is in agreement with Schofield's observation that the doubling time has increased after one passage through the spleen. Our data may suggest that during the first days after transplantation the excessive demand for differentiated cells in bone marrow is similar to that in spleen. 


\section{ACKNOWLEDGMENT}

The authors gratefully acknowledge the excellent technical assistance of Mr F. C. W. Luiten.

\section{REFERENCES}

Barnes, D.W.H., Ford, C.E. \& LoUTit, J.H. (1959) Greffes en série de moëlle osseuse homologue chez des souris irradiées. Sang, 30, 762 .

VAN BeKKUM, D.W. \& WeYZen, W.W.H. (1961) Serial transfer of isologous hematopoietic cells in irradiated hosts. Pathol. Biol. 9, 888 .

BENnetT, M. \& CUDKowicz, G. (1968) Hemopoietic progenitor cells of the mouse incapable of self-replication. Proc. Soc. exp. Biol. (N.Y.), 129, 99.

Blomgren, H. (1971) The role of bone marrow of X-irradiated mice in thymic recovery. Cell Tissue Kinet. $4,443$.

Cudkowicz, G., Upton, A.C., Smith, L.H., Gosslee, D.G. \& Hughes, W.L. (1964) An approach to the characterization of stem cells in mouse bone marrow. Ann. N.Y. Acad. Sci. 114, 571.

Daniel, Ch.W., De OME, K.B., Young, L.J.T., Blair, Ph.B. \& Faulkin, L.J. (1968) The in vivo life span of normal and preneoplastic mouse mammary glands: A serial transplantation study. Proc. nat. Acad. Sci. (Wash.), 61, 53.

Daniel, Ch.W. \& Young, L.J.T. (1971) Influence of cell division on an aging process. Life span of mouse mammary epithelium during serial propagation in vivo. Exp. Cell Res. 65, 27.

HaYflick, L. (1965) The limited in vitro lifetimes of human diploid cell strains. Exp. Cell Res. 37, 614.

Hayflick, L. \& MoORHEad, P.S. (1961) The serial cultivation of human diploid cell strains. Exp. Cell Res. $25,585$.

Holeckova, E. \& Cristofalo, V.J. (Eds.) (1970) Aging in Cell and Tisstie Culture. Plenum Press, New York.

KAY, H.E.M. (1965) How many cell generations? Lancet, ii, 418.

KROHN, P.L. (1962) Review lectures on senescence. II. Heterochromic transplantation in the study of aging. Proc. Roy. Soc. B, 157, 128.

LAJTha, L.G. \& Schofield, R. (1971) Regulation of stem cell renewal and differentiation: Possible significance in aging. Adv. gerontol. Res. 3, 131.

McCulloch, E.A. \& Till, J.E. (1964) Proliferation of hemopoietic colony-forming cells transplanted into irradiated mice. Radiat. Res. 22, 383.

SCHOFIELD, R. (1970) A comparative study of the repopulating potential of grafts from various haemopoietic sources: CFU repopulation. Cell Tissue Kinet. 3, 119.

Schofield, R. \& LAJTHA, L.G. (1969) Graft size considerations in the kinetics of spleen colony development. Cell Tissue Kinet. 2, 147.

Siminovitch, L., Till, J.E. \& McCulloch, E.A. (1964) Decline in colony-forming ability of bone marrow cells subjected to serial transplantation into irradiated mice. J. cell. comp. Physiol. 64, 23.

Vogel, H., Nifwisch, H. \& Matioli, G. (1968) The self renewal probability of hemopoietic stem cells. J. Cell Physiol. 72, 221.

Vos, O. (1967) Radiation sensitivity and post-irradiation repair of mouse lymphatic cells in vivo and in vitro. A study with the graft-versus-host reaction. Int.J. Radiat. Biol. 13, 317.

Vos, O. (1972a) Multiplication of haemopoietic colony-forming units (CFU) in mice after irradiation and bone marrow transplantation. Cell Tissue Kinet. 5, 341.

Vos, O. (1972b) Stem cell renewal in spleen and bone marrow of mice after repeated total-body irradiations Int.J. Radiat. Biol. 21, (in press). 